\title{
Study of Intermolecular Interactions Involved in Capillary GLC with Liquid Crystal Compounds as Stationary Phases
}

\author{
Felicia Spafiu ${ }^{1}$, Alice Mischie ${ }^{1}$, Adrian Beteringhe ${ }^{1,2}$, Titus Constantinescu ${ }^{1}$ and \\ Alexandru T. Balaban ${ }^{*}, 3$ \\ ${ }^{I}$ Institute of Physical Chemistry "Ilie Murgulescu" of the Romanian Academy, Splaiul Independentei 202, 060021 \\ Bucharest, Romania \\ ${ }^{2}$ University of South-East Europe "Lumina", Soseaua Colentina 64b, 021178 Bucharest, Romania \\ ${ }^{3}$ Texas A\&M University at Galveston, Department of Marine Sciences, 200 Seawolf Parkway, Galveston, TX 77551, \\ USA
}

\begin{abstract}
Abraham's equation was employed in order to investigate nonspecific intermolecular interactions involving liquid crystals. Several aromatic azo derivatives, proved to have liquid crystal behavior, were used as stationary phases in capillary gas chromatography. The polarizability, polarity, hydrophobicity and hydrogen-bond donor or acceptor characters were estimated using the Abraham solvation model. Calculations were based on the isothermal retention times for 25 compounds, in the temperature range between $70^{\circ}$ and $170^{\circ} \mathrm{C}$, both in the heating and the cooling mode. The descriptors $R, \pi, \Sigma \alpha, \Sigma \beta$, and $\log \mathrm{L}^{16}$ (Abraham parameters) were converted into orthogonal descriptors following Randić's procedure. The above-mentioned properties involve the $r, s, a, b$ and $l$ coefficients, respectively, which were determined by means of multilinear regression applied to the considered set. Following the orthogonalization procedure, the polarizability term $\left(r \mathrm{R}^{*}\right)$ was found to be statistically insignificant in the case of the studied compounds. Plots of these coefficients $v s$ temperature are discussed, in order to allow estimations regarding the retention mechanism and contributions of polar and Van der Waals interactions. The variation of properties (e.g. polarity, hydrogen-bond donor and hydrogen-bond acceptor character) with temperature was assigned to the changes in the molecular geometry of the compound used as stationary phase.
\end{abstract}

Keywords: Gas-liquid chromatography, Abraham's parameters, orthogonalization, liquid crystal.

\section{INTRODUCTION}

Intermolecular attractive forces can be specific or nonspecific. Supramolecular chemistry encompasses specific interactions involved in the design of new molecular architectures having pre-established properties in order to allow molecular associations similar to those occurring in biological media [1-5]. Some supramolecular self-assembly processes are also involved in the mesomorphic phenomena observed in the case of liquid crystals, leading to molecular aggregates with certain physical-chemical properties [6,7]. In fact, supramolecular complex formation consists in the selection and bonding of a substrate by a receptor molecule through directed physical interactions (electrostatic, hydrophobic, Van der Waals forces, or hydrogen bonds) [79]. Quantifying such interactions was attempted both by physical-chemical investigations and theoretical computational models [10-13].

Gas-liquid chromatography (GLC), which is currently used for the separation and detection of chemical

*Address correspondence to this author at the Texas A\&M University at Galveston, Department of Marine Sciences, 200 Seawolf Parkway, Galveston, TX 77551, USA; Tel: +1-409-741-4313; Fax: +1-409-740-4787; E-mail: balabana@tamug.edu compounds, proves to be useful also for investigating such properties of chemical compounds. The GLC retention time of a compound depends on its non-specific interaction with a liquid stationary phase, and therefore it characterizes both the solute and the stationary phase. Such interactions were investigated, and several solvation equations were developed [14] in order to allow calculations of properties of the stationary phases, starting from chromatographic retention data $[14,15]$. The present paper attempts to extend this kind of calculations involving liquid crystal compounds (1a-c and 2a, b) in order to estimate polarity, hydrophobicity and hydrogen-bond donor / acceptor characters, based on the Abraham solvation model [15] (eq 1).

$\log k=r \mathrm{R}+s \pi+a \Sigma \alpha+b \Sigma \beta+l \log \mathrm{L}^{16}+c$

where $k$ is the ratio between the adjusted retention time and the methane retention time; $\mathrm{R}, \pi, \Sigma \alpha, \Sigma \beta$, and $\log \mathrm{L}^{16}$ are the independent variables for the solute descriptors [14c, 15]. Each term of this equation defines a certain type of contribution to the total retention time: $r \mathrm{R}$ stands for the unshared or $\pi$ electron interactions, $s \pi$ is for dipole-dipole type interactions, $a \Sigma \alpha$ and $b \Sigma \beta$ are for $\mathrm{H}$-bond donor and $\mathrm{H}$ bond acceptor interactions, respectively, and $l \log \mathrm{L}^{16}$ is the contribution of dispersion forces $[14,15]$. The meaning of the five solute descriptors is as follows: $\mathrm{R}$ is for polarizability, $\pi$ for polarity, $\Sigma \alpha$ for H-bond-donor character, 
$\Sigma \beta$ for H-bond-acceptor character, and $\log L^{16}$ is the airhexadecane partition coefficient at $25^{\circ} \mathrm{C}$, which was used by Abraham as a measure of the dispersion interactions [15]. The coefficients $r, s, a, b$ correspond to the same properties in the case of the stationary phase (excepting $a$ and $b$ which by complementarity designate the proton acceptor and proton donor character, respectively, of the solvent [15]). The coefficient $l$ was assigned in previous communications $[14,15]$, to Van der Waals type interactions, and $c$ is a regression constant.

Previously synthesized azo-derivatives 1a-c and 2a, b were found to have liquid crystal properties [16-18]. These properties were investigated in the present paper through Abraham's equation (eq.1), because the temperature variations of coefficients in eq. 1 correspond to changes in the contributions to the retention time of the abovementioned interactions (namely polarity, H-bond donor/acceptor and dispersion interactions). Such studies have been communicated in the case of several amorphous polymers $[19,20]$. The temperature range was chosen so as to include the phase transition for each of the studied compounds, in order to monitor the changes in the properties of the liquid-crystal stationary phase. This novel approach could allow estimations of the mesomorphic phase properties, which cannot be measured by other means.

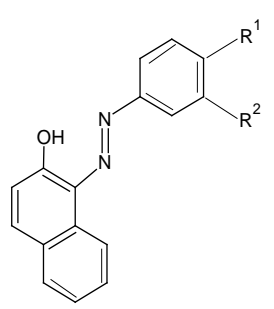

1a-c

a: $\mathrm{R}^{1}=\mathrm{R}^{2}=\mathrm{H}$

b: $\mathrm{R}^{1}=\mathrm{OCH}_{3} ; \mathrm{R}^{2}=\mathrm{H}$

c: $\mathrm{R}^{1}=\mathrm{R}^{2}=\mathrm{OCH}_{3}$

\section{MATERIALS AND METHODOLOGY}

The synthesis of compounds 1a-c and 2a, $\mathbf{b}$, thermal data by differential scanning calorimetry (DSC) analysis, the preparation of the capillary columns using compounds 1a-c and $\mathbf{2 a}, \mathbf{b}$ as stationary phases, and the measurement of GC retention times for 25 solutes (Table $\mathbf{1}$ ) was performed in our previous papers [16-18]. For comparison, similar experiments were described [18] using OV-25 (which has a methyl-phenyl-polysiloxane structure) as stationary phase.

The orthogonalization procedure was developed by M. Randić [21-23] for multilinear regressions. For a considered descriptor set, one of the descriptors is considered orthogonal from the beginning. In the present case, this will be the $\log L^{16}$ descriptor. The second orthogonal descriptor will be the residual between the second and the first descriptor (Res 2/1). The third orthogonal descriptor is obtained by correlating the third descriptor against the first, which gives the corresponding residual, Res 3/1. This residual, while orthogonal to the first descriptor, will not be orthogonal to the second orthogonal descriptor. To make it orthogonal also to the second descriptor, one considers the regression of Res $3 / 1$ against Res $2 / 1$. The residual of this regression, Res $3 / 2$, is the third orthogonal descriptor. In the case of additional descriptors the outlined procedure is continued until all the descriptors are made orthogonal.

The geometry optimization and estimation of the lowest energy conformation for the $E$ and $Z$ isomers of structure 1a were performed by using Marvin's molecular modeling tool [24].

\section{RESULTS AND DISCUSSION}

In earlier communications [16-18], results of DSC analysis and GC proved that compounds $\mathbf{1 a - c}$ and $\mathbf{2 a}$, b exhibit liquid crystal behavior, with differences between the heating mode and the cooling mode. Abraham's equation was applied to the retention times of a set of 25 compounds, measured at temperatures between $70^{\circ} \mathrm{C}$ and $170^{\circ} \mathrm{C}$ [17]. The phase transition range of the studied compounds was determined both by GC and by DSC analysis [18].

The values of the Abraham parameters, $R, \pi, \Sigma \alpha, \Sigma \beta$, $\log L^{16}$ (see eq 1) for the solutes are presented in Table 1 .

Table 1. Values of Abraham's Parameters $(R, \pi, \Sigma \alpha, \Sigma \beta$, $\left.\log L^{16}\right)[14,15]$

\begin{tabular}{|c|c|c|c|c|c|}
\hline Solute & $\mathbf{R}_{\text {calc }}$ & $\boldsymbol{\pi}$ & $\sum \boldsymbol{\alpha}$ & $\sum \boldsymbol{\beta}$ & $\log \boldsymbol{L}^{\mathbf{1 6}}$ \\
\hline \hline n-decane & 0.000 & 0.00 & 0.00 & 0.00 & 4.69 \\
\hline n-undecane & 0.000 & 0.00 & 0.00 & 0.00 & 5.19 \\
\hline n-dodecane & 0.000 & 0.00 & 0.00 & 0.00 & 5.70 \\
\hline n-tetradecane & 0.000 & 0.00 & 0.00 & 0.00 & 6.71 \\
\hline n-hexadecane & 0.000 & 0.00 & 0.00 & 0.00 & 7.71 \\
\hline n-hexanol & 0.210 & 0.42 & 0.37 & 0.48 & 3.61 \\
\hline n-heptanol & 0.202 & 0.42 & 0.37 & 0.48 & 4.12 \\
\hline n-octanol & 0.197 & 0.42 & 0.37 & 0.48 & 4.62 \\
\hline n-nonanol & 0.191 & 0.42 & 0.37 & 0.48 & 5.12 \\
\hline n-decanol & 0.191 & 0.42 & 0.37 & 0.48 & 5.63 \\
\hline n-undecanol & 0.188 & 0.42 & 0.37 & 0.48 & 6.13 \\
\hline n-dodecanol & 0.180 & 0.42 & 0.37 & 0.48 & 6.64 \\
\hline tetrahydrolinalool & 0.167 & 0.30 & 0.31 & 0.60 & 5.27 \\
\hline tetrahydrolavandulol & 0.142 & 0.36 & 0.33 & 0.56 & 5.24 \\
\hline tetrahydrogeraniol & 0.175 & 0.36 & 0.33 & 0.56 & 5.31 \\
\hline methyl nonanoate & 0.054 & 0.60 & 0.00 & 0.45 & 5.19 \\
\hline methyl decanoate & 0.049 & 0.60 & 0.00 & 0.45 & 5.41 \\
\hline methyl undecanoate & 0.050 & 0.60 & 0.00 & 0.45 & 6.00 \\
\hline methyl dodecanoate & 0.046 & 0.60 & 0.00 & 0.45 & 6.55 \\
\hline methyl tridecanoate & 0.046 & 0.60 & 0.00 & 0.45 & 7.09 \\
\hline octyl acetate & 0.029 & 0.60 & 0.00 & 0.45 & 5.36 \\
\hline nonyl acetate & 0.040 & 0.60 & 0.00 & 0.45 & 5.68 \\
\hline decyl acetate & 0.030 & 0.60 & 0.00 & 0.45 & 6.37 \\
\hline tetrahydrolinalyl acetate & 0.020 & 0.57 & 0.00 & 0.47 & 5.41 \\
\hline tetrahydrolavandulyl acetate & 0.020 & 0.57 & 0.00 & 0.47 & 5.41 \\
\hline & & & & \\
\hline & & & \\
\hline
\end{tabular}


In the present case some of the parameters are highly interrelated so that the orthogonalization procedure was necessary for obtaining meaningful coefficients. The corresponding orthogonal parameters calculated for the considered solute set are given in Table $\mathbf{2}$.

Table 2. Values of the Orthogonal Parameters $\left(R^{*} \pi,{ }^{*} \Sigma \alpha, *\right.$ $\left.\Sigma \beta,{ }^{*} \log L^{16 *}\right)$ Used in the Regressions

\begin{tabular}{|c|c|c|c|c|c|}
\hline Solute & $\boldsymbol{R}^{*}$ & $\boldsymbol{\pi}^{*}$ & $\boldsymbol{\Sigma} \boldsymbol{\alpha}^{*}$ & $\boldsymbol{\Sigma} \boldsymbol{\beta}^{*}$ & $\log \boldsymbol{L}^{\mathbf{1 6 *}}$ \\
\hline \hline n-decane & -0.0038 & -0.410 & -0.039 & -0.139 & 4.69 \\
\hline n-undecane & -0.0024 & -0.402 & -0.029 & -0.116 & 5.19 \\
\hline n-dodecane & -0.0009 & -0.395 & -0.019 & -0.093 & 5.70 \\
\hline n-tetradecane & 0.0020 & -0.380 & 0.001 & -0.047 & 6.71 \\
\hline n-hexadecane & 0.0049 & -0.364 & 0.022 & -0.002 & 7.71 \\
\hline n-hexanol & 0.0114 & -0.006 & 0.086 & -0.012 & 3.61 \\
\hline n-heptanol & 0.0053 & 0.002 & 0.097 & 0.011 & 4.12 \\
\hline n-octanol & 0.0013 & 0.009 & 0.107 & 0.033 & 4.62 \\
\hline n-nonanol & -0.0028 & 0.017 & 0.117 & 0.056 & 5.12 \\
\hline n-decanol & -0.0012 & 0.024 & 0.127 & 0.079 & 5.63 \\
\hline n-undecanol & -0.0028 & 0.032 & 0.137 & 0.102 & 6.13 \\
\hline n-dodecanol & -0.0097 & 0.040 & 0.147 & 0.125 & 6.64 \\
\hline tetrahydrolinalool & 0.0161 & -0.101 & -0.212 & 0.270 & 5.27 \\
\hline tetrahydrolavandulol & -0.0249 & -0.042 & -0.083 & 0.185 & 5.24 \\
\hline tetrahydrogeraniol & 0.0083 & -0.041 & -0.082 & 0.188 & 5.31 \\
\hline methyl nonanoate & 0.0133 & 0.198 & -0.040 & -0.102 & 5.19 \\
\hline methyl decanoate & 0.0087 & 0.201 & -0.036 & -0.092 & 5.41 \\
\hline methyl undecanoate & 0.0110 & 0.210 & -0.024 & -0.065 & 6.00 \\
\hline methyl dodecanoate & 0.0084 & 0.218 & -0.013 & -0.041 & 6.55 \\
\hline methyl tridecanoate & 0.0102 & 0.226 & -0.002 & -0.016 & 7.09 \\
\hline octyl acetate & -0.0115 & 0.200 & -0.036 & -0.094 & 5.36 \\
\hline nonyl acetate & 0.0003 & 0.205 & -0.030 & -0.080 & 5.68 \\
\hline decyl acetate & -0.0076 & 0.216 & -0.016 & -0.048 & 6.37 \\
\hline tetrahydrolinalyl acetate & -0.0168 & 0.171 & -0.090 & -0.050 & 5.41 \\
\hline tetrahydrolavandulyl acetate & -0.0168 & 0.171 & -0.090 & -0.050 & 5.41 \\
\hline
\end{tabular}

The orthogonalization method was introduced by Randić [21-23] in order to solve the instability problem for regression coefficients as the result of the interrelation of the parameters. Interrelated descriptors contain a common amount of information, but eliminating one of these descriptors may cause losing some other important information. With respect to this, the term of "orthogonal descriptor" refers to the part of a descriptor that does not correlate with the other descriptors. When two descriptors are involved, an orthogonal parameter results as the residual between the second and the first descriptor. The procedure is general and it can be applied regardless of how much or how little the descriptors are interrelated. Since the orthogonal descriptor is obtained as a linear combination of the original descriptors, it will bring the same information as the original ones, eliminating the information which is common to the other descriptors. The Pearson coefficient is not changed by the orthogonalization procedure, the regression coefficients became stable, and interpretations are allowed [21]. The orthogonalization order of descriptors was established by retro-regression [22]. The concept of retro-regression, or backward stepwise regression, starts from the regression equation as the solution and searches for an ordering of descriptors. Thus one searches the descriptor that makes the least significant contribution. When identified, such a descriptor will be eliminated as the least important. The process continues with a search for the next least important descriptor, till all but the last of the descriptors is eliminated stepwise.

It can be observed that the last columns in Tables $\mathbf{1}$ and $\mathbf{2}$ are identical, because the orthogonalization started with the descriptor $\log L^{16}$.

Tables 3 and 4 show the correlation matrices for the original Abraham parameters $R, \pi, \Sigma \alpha, \Sigma \beta, \log L^{16}$ and for the derived orthogonal descriptors $\mathrm{R}^{*}, \pi^{*}, \Sigma \alpha^{*}, \Sigma \beta^{*}, \log L^{16^{*}}$.

Table 3. Correlation Matrix of Abraham Parameters $(R, \pi$, $\Sigma \alpha, \Sigma \beta, \log L^{16}$ ) for the Considered Solutes

\begin{tabular}{|c|c|c|c|c|c|}
\hline & $\mathbf{R}_{\text {calc }}$ & $\boldsymbol{\pi}$ & $\sum \boldsymbol{\alpha}$ & $\sum \boldsymbol{\beta}$ & $\log \mathbf{L}^{16}$ \\
\hline \hline $\mathrm{R}_{\text {calc }}$ & 1 & & & & \\
\hline$\pi$ & 0.187 & 1 & & & \\
\hline$\sum \alpha$ & 0.974 & 0.009 & 1 & & \\
\hline$\sum \beta$ & 0.627 & 0.822 & 0.500 & 1 & \\
\hline $\log \mathrm{L}^{16}$ & -0.428 & -0.061 & -0.402 & -0.255 & 1 \\
\hline
\end{tabular}

Table 4. Correlation Matrix of the Orthogonal Parameters $\left(R^{*}, \pi^{*}, \Sigma \alpha^{*}, \Sigma \beta^{*}, \log L^{16 *}\right)$ Used in the Regressions

\begin{tabular}{|c|c|c|c|c|c|}
\hline & $\mathbf{R}_{\text {calc }}^{*}$ & $\Pi^{*}$ & $\sum \boldsymbol{\alpha}^{*}$ & $\sum \boldsymbol{\beta}^{*}$ & $\log \mathbf{L}^{16 *}$ \\
\hline \hline $\mathrm{R}_{\text {calc }}$ & 1 & & & & \\
\hline$\pi^{*}$ & $2.20 \mathrm{E}-4$ & 1 & & & \\
\hline$\sum \alpha^{*}$ & $-2.50 \mathrm{E}-5$ & $1.24 \mathrm{E}-5$ & 1 & & \\
\hline$\sum \beta^{*}$ & $6.91 \mathrm{E}-5$ & $6.72 \mathrm{E}-6$ & $6.31 \mathrm{E}-6$ & 1 & \\
\hline $\log \mathrm{L}^{16 *}$ & $-4.50 \mathrm{E}-4$ & $1.46 \mathrm{E}-5$ & $3.47 \mathrm{E}-6$ & $1.69 \mathrm{E}-5$ & 1 \\
\hline
\end{tabular}

In the case of the stationary phases studied (1a-c, and $\mathbf{2 a}$, b) and also for $\mathbf{O V}-25, R^{*}$ was found to be statistically insignificant, so that it was no more included in the regression equations.

The coefficients $s, a, b$ and $l$ obtained by the multilinear regression for the considered set are given in Table S1 (Supplementary Material). Pearson coefficients $\left(R^{2}\right)$ of these regressions were between 0.93 and 0.99 (Table S1 in Supplementary Material).

These results can be summarized as plots of $\log k$ (for decane and decanol) and of the determined Abraham coefficients, exemplified in Fig. (1), for stationary phases 1a and OV-25. The diagrams in Fig. (1) highlight some 


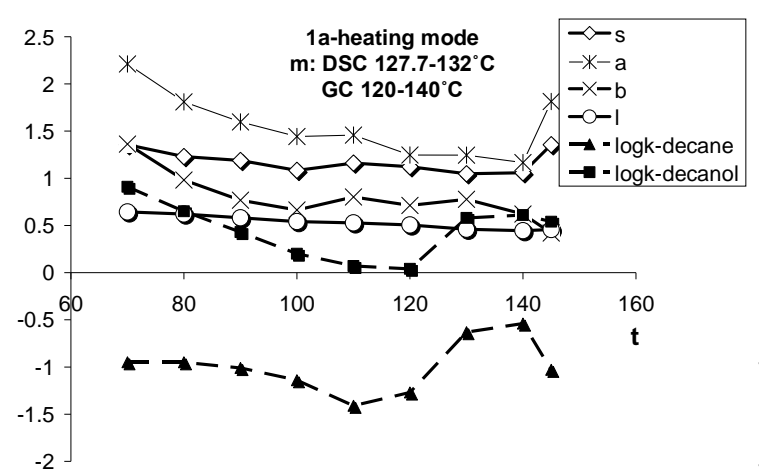

C

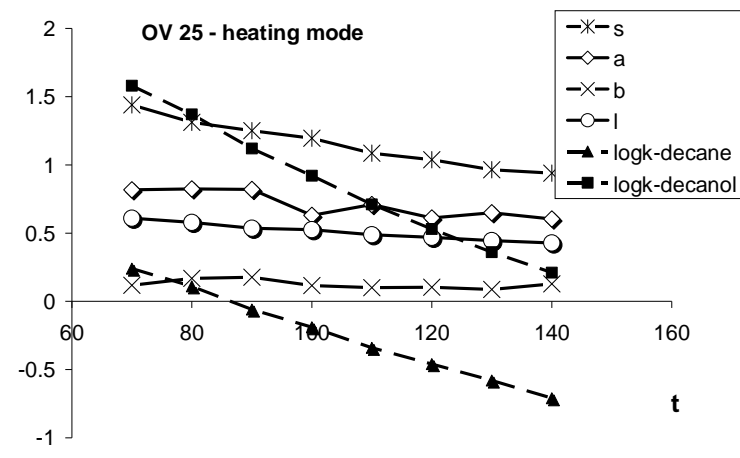

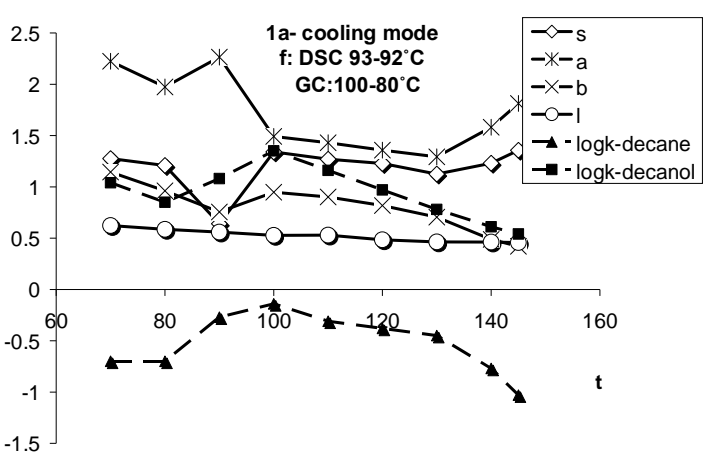

$\mathrm{D}$

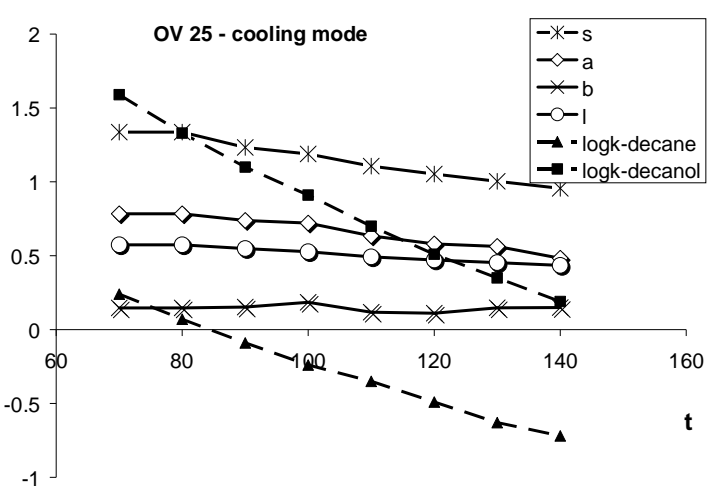

Fig. (1). Plots of the $\log k$ of decane and decanol and of the $s, a, b, l$ coefficients $v s$ temperature for the studied stationary phase 1a (A, B) compared to OV-25 (C, D).

interesting aspects regarding the solute-stationary phase association mechanism for estimation of the dipole-dipole, $\mathrm{H}-$ bond and dispersion interactions for each case.

For the stationary phase 1a, the retention diagrams confirm the anisotropic behavior, with differences between the retention times during the heating mode $v s$ the cooling mode. The same differences could be observed in the case of the polarity $(s)$ and also of the hydrogen bonding ability ( $a$, $b$ ), but not in the case of the $l$ coefficient of the dispersion interaction descriptor $\left(\log L^{16}\right)$. The last parameter has a linear variation $v s$ temperature, identical for the heating and the cooling modes.

For the OV-25 stationary phase, which is not a crystalline liquid, the above mentioned differences between heating and cooling were not observed. According to several studies on other amorphous liquid stationary phases $[19,20]$, intermolecular forces expressed by the coefficients $s, a, b$ and $l$ were found to be linearly decreasing with increasing temperatures.

In the case of compounds 1a-c and $\mathbf{2 a - b}$, the shape of the retention time plots is changed in the phase transition region, due mainly to bulk absorption, as reported in the literature [25]. One can note that the melting and freezing intervals appearing on the retention diagrams are different, namely more extended towards those determined by the DSC method, indicating changes in the solute binding which are not correlated with the phase transition phenomena. Molecular shape is supposed to determine the observed changes in the solute absorption, by modifying the dipole moment and accessibility of polar groups for hydrogen bonding.

The polar groups in structures 1a-c and 2a, b are represented by the phenolic hydroxyl and methoxy groups. Molecular geometry appears to be controlled by the $E-Z$ (syn-anti) isomerism influencing the steric hindrance. For the existing anti $(E)$ isomer, aromatic rings are not sterically hindered, so that a planar molecular shape will be expected. In the case of a hypothetical syn $(Z)$ isomer, steric hindrance would cause the non-planarity of the two aromatic moieties (Fig. 2).

In the case of the $E$ isomer, intramolecular hydrogen bonding will occur due to a favorable position of the lone pair of an azo nitrogen towards the hydroxyl group. Marvincomputed distances between the hydroxyl proton and the corresponding nitrogen atom are $2.58 \AA$ for the $E$ isomer and $3.94 \AA$ for $Z$, respectively, so that internal chelate formation may be expected in the first case [26].

For the studied azo-derivatives, the synthesis afforded the lower-energy $E$ isomer [16], corresponding to the intense color of these compounds both in solid state and in solution. 


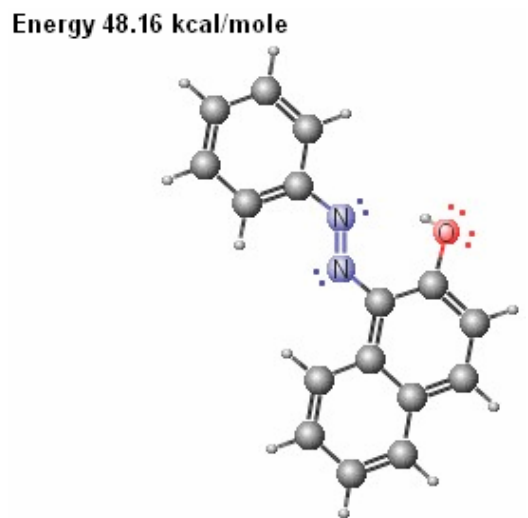

(a) $E$-isomer

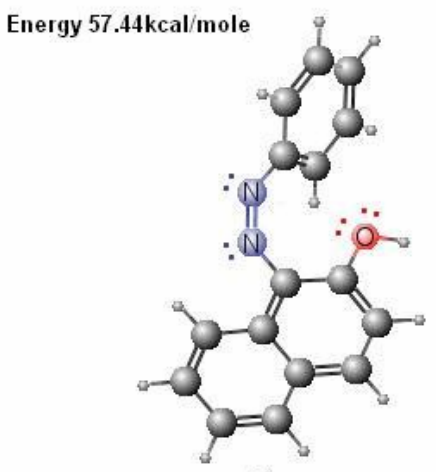

(b) Z-isomer

Fig. (2). Geometry optimization for the structure 1a. (Using MarvinSketch Calculator Plugin [24]).

Additionally, an intramolecular hydrogen bond $(\mathrm{OH} \ldots \mathrm{N}=\mathrm{N})$ was found by NMR investigation for all 1a-c and 2a, b derivatives [16]. On heating, the low-energy configuration shown in Fig. (2a) will change, and an interconversion between the $E$ and $Z$ isomers may be promoted by temperature variation. One can note that the breaking of the internal chelate will result in an enhancement of hydrogenbond properties of the stationary phase, depending on the steric availability of the donor/acceptor groups. Indeed, this is confirmed by the positive slope for the variation of the $a$ and $s$ coefficients of compound 1a at temperatures around $140^{\circ} \mathrm{C}$ (Fig. 1). More precise correlations between the temperature variation of the stationary phase properties and the corresponding molecular geometry will be approached in future work.

\section{CONCLUSIONS}

Property determinations were attempted for five aromatic azo-derivatives (1a-c, 2a, 2b) exhibiting liquid crystal behavior. Polarity, hydrophobicity and H-bond donor/acceptor character were estimated using Abraham's solvation model. The temperature range was chosen so as to include the mesomorphic phase transition for each of the studied compounds, in order to monitor the changes in the stationary phase properties. Plots of Abraham's coefficients $(s, a, b, l)$ vs temperature have confirmed the liquid crystal character of the studied compounds, previously detected by DSC measurements. The observed changes in the mentioned properties were attributed to the corresponding change of the molecular shape of the stationary phase. In the case of the studied azo-derivatives, such geometrical rearrangements may be related to the $E-Z$ isomerism and to the relative rotation of the two aromatic rings.

\section{ACKNOWLEDGEMENTS}

This research was performed within the program "Coordinative and Supramolecular Chemistry" of the "Ilie Murgulescu" Institute of Physical Chemistry, financed by the Roumanian Academy.

\section{CONFLICT OF INTEREST}

The authors confirm that this article content has no conflicts of interest.

\section{SUPPLEMENTARY MATERIAL}

Supplementary material is available on the publisher's web site along with the published article.

\section{REFERENCES}

[1] Wolf KL, Harms $\mathrm{H}$. The state of arrangement of molecules in liquids. Z Phys Chem B 1937; 46: 237-86.

[2] Lehn JM. Design of organic complexing agents. Strategies towards properties. Struct Bonding 1973; 16: 1-69.

[3] Lehn JM. Supramolecular chemistry; concepts and perspectives. Weinheim: Wiley-VCH 1995.

[4] Lehn JM. Supramolecular chemistry; scope and perspectives. Molecules, supermolecules, and molecular devices (Nobel Lecture). Angew Chem Int Ed Engl 1988; 27: 89-112.

[5] Ehlrich P. Studies on immunity. New York: Wiley 1906.

[6] Lehn JM, Simon J, Wagner J. Mesomolecules. Polyaza-polyoxa macrocyclic systems. Angew Chem Int Ed Engl 1973; 12: 578-9.

[7] Lehn, JM. Cryptates, inclusion complexes of macropolycyclic receptor molecules. Pure Appl Chem 1978; 50: 871-92.

[8] Weber E, Vögtle F. Progress in crown ether chemistry. Kontakte 1981; 1: 26-9.

[9] Lehn JM. Perspectives in supramolecular chemistry - From molecular recognition towards molecular information processing and self-organization. Angew Chem Int Ed Engl 1990; 29: 130419.

[10] Fitzmaurice RJ, Gaggini F, Srinivasan N, Kilburn JD. Carboxylate binding in polar solvents using pyridyl guanidinium salts. Org Biomol Chem 2007; 5: 1706-14.

[11] Mati IK, Cockroft SK. Molecular balances for quantifying noncovalent interactions. Chem Soc Rev 2010; 39: 4195-205.

[12] Raevsky A. Quantification of non-covalent interactions on the basis of thermodynamic hydrogen bond parameters. J Phys Org Chem 1997; 10: 405-13.

[13] Fujita T, Nishioka T, Nakajima M. Hydrogen-bonding parameter and its significance in quantitative structure-activity studies. J Med Chem 1977; 20: 1071-81.

[14] (a) Patte F, Etcheto M, Laffort P. Solubility factors for 240 solutes and 207 stationary phases in gas-liquid chromatography. Anal Chem 1982; 54: 2239-47. (b) Laffort P, Chauvin F, Dallos A, Callegari P. Solvation parameters. Part 1. Mutual improvements of several approaches and determination of two first sets of optimized values. J Chromatogr A 2005; 1100: 90-107. (c) Abraham MH, Whitting G. Hydrogen bonding. Part 13. A new method for the characterization of glc stationary phases - the Laffort data set. J Chem Soc Perkin Trans 1 1990; 2: 1451-60.

[15] Abraham MH. Scales of solute hydrogen bonding, their construction and application to physicochemical and biochemical processes. Chem Soc Rev 1993; 73-83.

[16] Luca C, Spafiu F, Caproiu MT, et al. Gas chromatographic studies using glass capillary columns with crown ether type stationary phases. Rev Roum Chim 1998; 43: 287-93. 
[17] Spafiu F, Tudor E, Popa V, Constantinescu T, Stanciuc A, Luca C. Gas chromatographic studies using glass capillary columns with naphthyl-azobenzo-crown ethers and related compounds as stationary phases. Rev Roum Chim 2001; 46: 19-30.

[18] Spafiu F, Manolescu G, Radulescu S, Stancioiu C, Constantinescu T. Gas chromatographic studies using glass capillary columns with naphthyl azacrown ethers and related compounds as stationary phases. Rev Roum Chim 2001; 46: 229-41.

[19] Tello AM, Lebrón-Aguilar R, Quintanilla-López JE, Pérez-Parajón JM, Santiuste JM. Application of the solvation parameter model to poly (methylcyanopropylsiloxane) stationary phases. J Chromatogr A 2006; 1122: 230-41.

[20] Santiuste JM. Temperature effect on the characteristic solutesolvent retention interactions, calculated with Abraham's solvation model, for 16 GLC stationary phases. Anal Chim Acta 1998; 377: $71-83$.
[21] Randić M. Resolution of ambiguities in structure-property studies by use of orthogonal descriptors. J Chem Inf Comput Sci 1991; 31: 311-20.

[22] Randić M. Regression. Another important multivariate regression improvement. J Chem Inf Comput Sci 2001; 41: 602-6.

[23] Randić M. Orthosimilarity. J Chem Inf Comput Sci 1996; 36: 1092-7.

[24] Marvin Applets, Chem Axon Version 5.6.0.5. Available at: http://www.chemaxon.com

[25] Kunaver M, Zadnik J, Planinsek O, Srcic S. Inverse gas chromatography: a different approach to characterization of solids and liquids. Acta Chim Slov 2004; 51: 373-94.

[26] Arunan E, Desiraju GR, Klein RA, et al. Definition of the hydrogen bond. Pure Appl Chem 2011; 83: 1637-41.

Received: June 22, 2012

Revised: July 5, 2012

Accepted: August 11, 2012

(C) Spafiu et al.; Licensee Bentham Open.

This is an open access article licensed under the terms of the Creative Commons Attribution Non-Commercial License (http://creativecommons.org/licenses/by-nc/ 3.0/) which permits unrestricted, non-commercial use, distribution and reproduction in any medium, provided the work is properly cited. 